\title{
Lab-on-a-Chip Platforms for Disease Detection and Diagnosis
}

Ziya Isiksacan', Mustafa Tahsin Guler', Ali Kalantarifard', Mohammad Asghari', and Caglar Elbuken'

'Institute of Materials Science and Nanotechnology, National Nanotechnology Research Center (UNAM), Bilkent University, Ankara, Turkey

${ }^{2}$ Department of Physics, Kirikkale University, Kirikkale, Turkey

\subsection{Introduction}

The roots of understanding the liquid behaviors date back to centuries ago. Initial examples cover the studies by Hippocrates (400 BC) and Galen (200 AD) who performed the colorimetric analysis of body fluids like urine to monitor human physiology [1]. With the advancement of microfabrication methods and tools, dexterous handling of liquids in small quantities and development of small fluidic components (valves, pumps, etc.) were witnessed after the 1950s. Seminal studies include the development of miniaturized gas chromatography chip [2] and ink-jet printing technology [3]. In 1990, Andreas Manz proposed the concept of the micro-total analysis systems ( $\mu \mathrm{TAS})$, addressing the possibility that miniaturized integrated systems can be developed to perform biochemical analysis with much lower sample volumes [4]. Then, microfluidics has emerged as a discipline where very low volumes of fluids are manipulated and studied, employing structures in micron length scales. Conventional photolithographic techniques used for silicon and glass wafers in microelectronics and MEMS have been inherited for the fabrication of microfluidic chips. Polydimethylsiloxane (PDMS) was utilized by George Whitesides for soft lithography introducing rapid fabrication of microfluidic devices [5].

Years of research have shown that this elastomeric, optically transparent, porous, and biocompatible material is suitable for many microfluidic applications. The utmost goal of $\mu$ TAS is to integrate laboratory tasks on a single chip, namely, lab-on-a-chip (LOC). The LOC systems, once successfully established, 
have the benefits of low cost, automation, high throughput, multiplexing, and portability. It is also critical to minimize the number of off-chip components such as power sources and pumps to turn microfluidic systems into truly LOC systems. This distinction is especially noteworthy for disease diagnostics at the point of care (POC) where the ASSURED criteria are coined by the World Health Organization (WHO) $[6,7]$. The acronym stands for "affordable, sensitive, specific, user-friendly, rapid and robust, equipment free, and deliverable to end-user." Various body fluids such as blood, urine, or saliva can be used to look for specific biomarkers. The most commonly investigated biomarkers include proteins (especially for immunoassays), cells (circulating tumor cells, red blood cells, platelets, etc.), nucleic acids (DNA, RNA, etc.), metabolites (glucose, lactate, etc.), and ionic chemicals (sodium, potassium, etc.) [8, 9]. Depending on the type of biomarker as well as the working mechanism of the LOC platform, a number of detection principles can be proposed. The most preferred ones include optical detection (colorimetric, spectroscopic, plasmonic, fluorescence) and electrical detection (electrochemical, piezoelectric, impedimetric, potentiometric). This chapter discusses different LOC platforms that are employed for the detection and diagnosis of diseases and are categorized into six groups: continuous flow, paper-based, microdroplet, digital microfluidic (DMF), compact disc-based, and wearable platforms. Each platform is explained in the following subsections, and seminal studies as well as the state of the art are discussed. Finally, a future perspective and outlook are provided.

\subsection{Continuous Flow Platforms}

Continuous flow microfluidic platforms are the conventional microfluidic diagnostic systems where a single-phase fluid flow is controlled inside microfluidic channels. The initial examples of continuous flow systems were fabricated on glass using conventional microfabrication techniques. The patterned glass layer is bonded with another glass piece, forming micrometer-scale delicate structures. After the adaptation of polymers, mostly PDMS, by the microfluidics community, the field witnessed an explosion of research output all across the world. The integrated platforms require on-chip pumps, valves, mixers, and sensors. There has been considerable effort on developing these individual components as summarized in several review articles [10-14].

One of the earliest examples of continuous flow LOC detection systems is the DNA analysis system [15]. It is designed for microfluidic DNA gel electrophoresis and contains sample preparation units such as sample metering, mixing, and heating, as well as integrated fluorescence optical detection unit. The system has a hybrid silicon/glass structure that is defined by photolithographic techniques. The system can meter a specific volume of DNA fragment 
solution, run it through an electrophoretic separation channel, and perform fluorescent detection of the separated DNA segments.

With the wide use of PDMS in microfluidics community, the number of continuous flow diagnostic microfluidic systems has inflated. A cornerstone for PDMS-related devices was the development of elastomeric valve. Later, these microvalves have been integrated on a single device at a highly scalable level that led to very-large-scale integrated valve-based PDMS microfluidic devices [16]. The fundamental technology behind these systems is the development of two-layer microfluidic systems that are fabricated using soft lithography. One of the layers is utilized as the fluidic layer whereas the second layer is designed as the control layer. The two layers were separated using thin PDMS elastomeric valves that can be actuated using increased pressures to seal the crossing fluid layer.

These two-layer devices allowed exquisite control on the fluid motion along a microchannel network that opened up a plethora of applications. An exemplary system is shown in Figure 8.1, which performs on-chip DNA isolation from whole bacteria [17]. The system is composed of 54 elastomeric valves and can perform parallel processing in three lines. First, a single cell is isolated from a cell solution. Then, cell lysis is performed using a rotary mixer, which is composed of a ring-shaped channel with three sequential valves. The operation of these three valves in a certain sequence results in peristaltic pumping of the fluid, which is utilized as a rotary mixer, enhancing diffusion-limited processes for such biochemical applications. After cell lysis, the mixture is transferred to a DNA affinity column for purification and then transferred to the system outlet. As seen in Figure 8.1, in an area of $2 \mathrm{~cm} \times 2 \mathrm{~cm}$, the system integrates a complicated network of two-layer PDMS channels and achieves sophisticated fluidic operations that can be programmed and scaled up in parallel. Such high-level integration of fluid control at microfluidic scale was a revolution in the continuous flow systems and triggered several other advanced detection and diagnostic devices such as fluorescence-activated cell sorting [18] and single-cell enzyme screening [19].

Nucleic acid detection is a very sensitive and specific strategy that has been successfully implemented on continuous microfluidic systems. Another challenge in the disease diagnosis is the detection of rare cells. Identification of cells in a given solution, for example, blood, is performed using flow cytometers. There have been several remarkable examples of continuous flow cytometry systems [20]. However, detection of rare cells poses another level of complexity since these applications require cell purification and high-throughput analysis systems. Detection of circulating tumor cells (CTCs) is an excellent example of rare cell detection, which is critical for early diagnosis of cancer.

Continuous flow LOC systems provide a very suitable platform for detection of CTCs. An outstanding example of such systems is developed by Prof. Toner's group. They demonstrated a microfluidic system that is composed of 
(a)

(b)
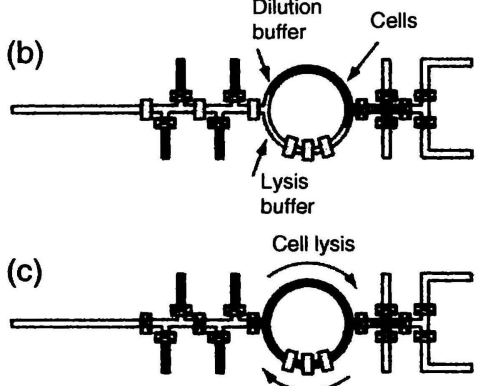

(d)

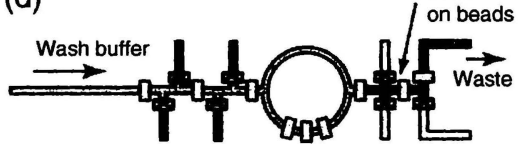

(e)

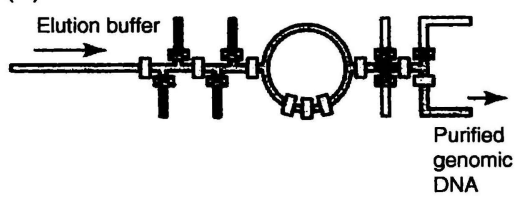

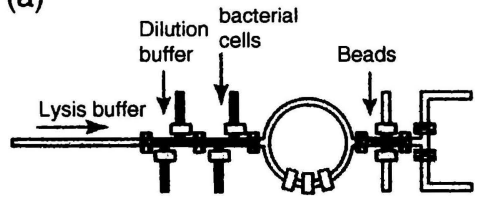

(f)

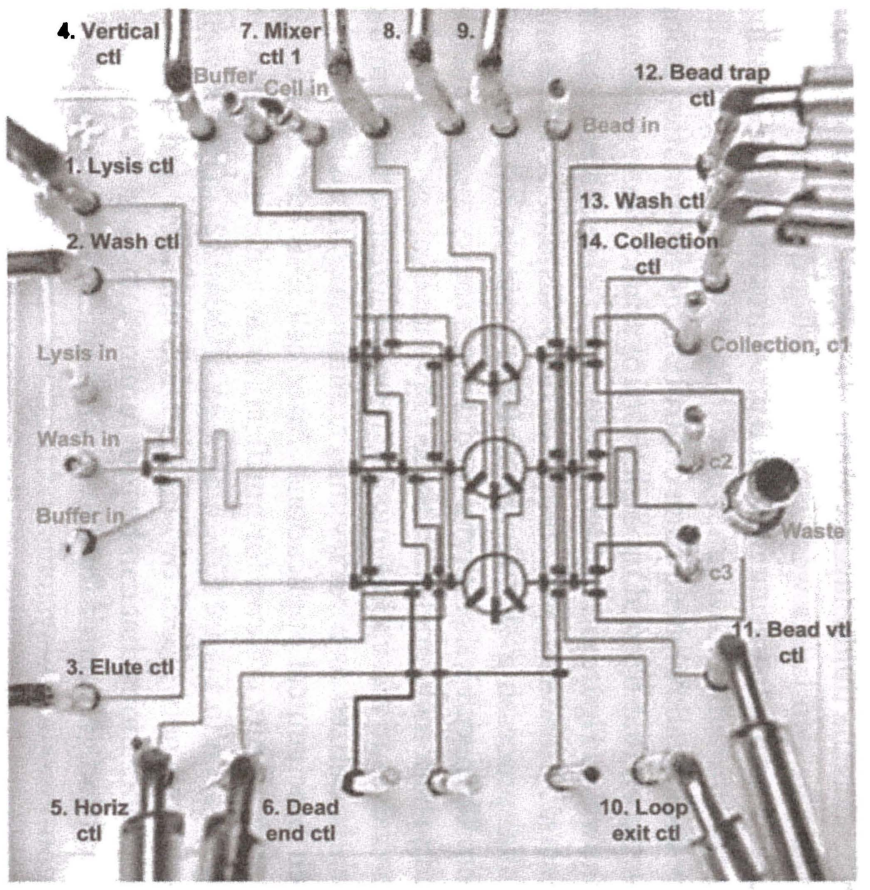

Figure 8.1 Automated on-chip DNA purification. (a-e) The workflow summarizing the on-chip DNA isolation protocol. (f) Picture of the parallel DNA purifier system that has a two-layer structure for fluid flow and valve actuation. Fluid channels and control channels have widths of 100 and $200 \mu \mathrm{m}$, respectively. Source: Hong et al. [17]. Reproduced with permission of Nature Publishing Group. 


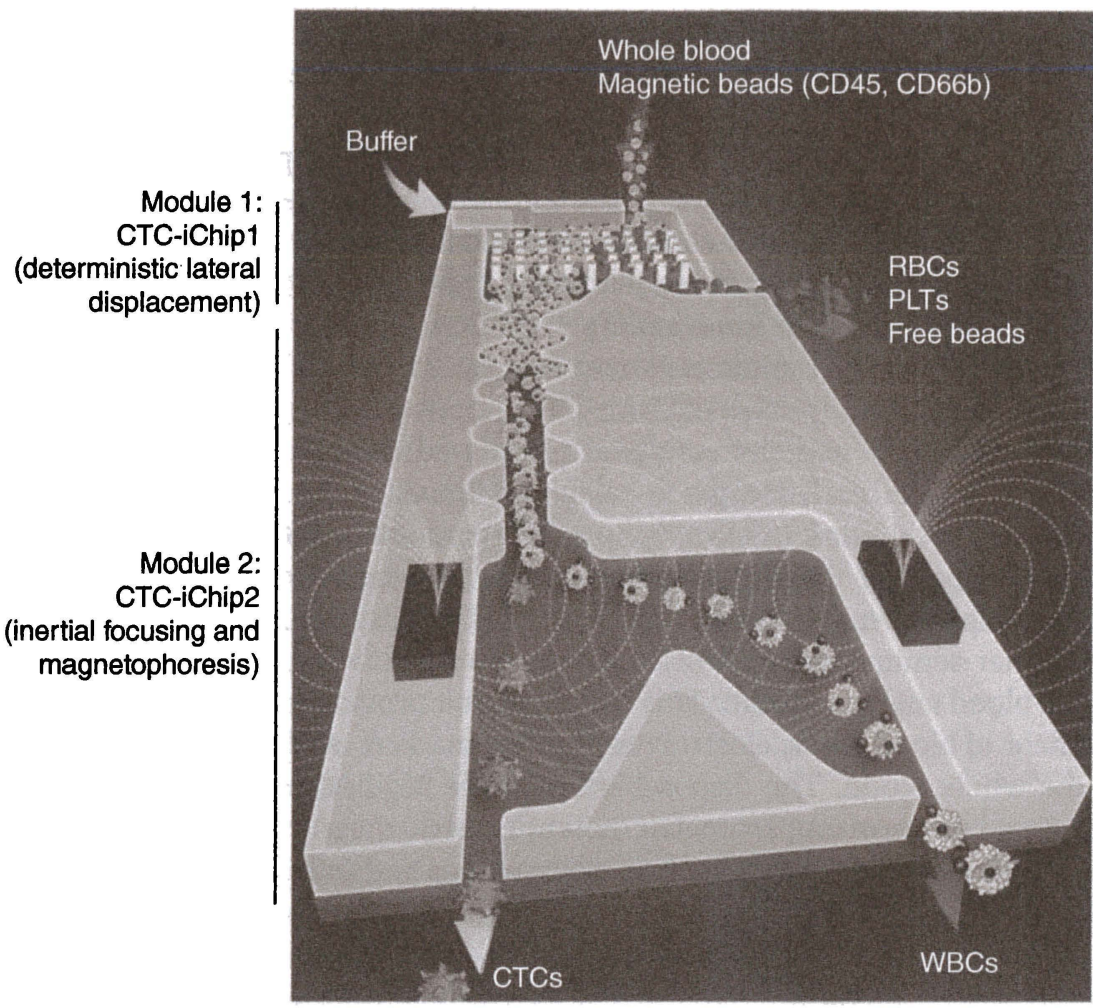

Figure 8.2 CTC isolation chip. Whole blood mixed with antibody-tagged magnetic beads is sent to the channel in parallel with buffer solution. Deterministic lateral displacement compartment of the chip enables the separation of white blood cells (WBCs) and CTCs from the whole blood. Then, WBCs and CTCs are brought on a single line after passing asymmetric focusing elements utilizing inertial microfluidics. Finally, magnetic force is applied over centrally aligned cells that force magnetically labeled CTCs to a different outlet, completing the isolation process. Source: Karabacak et al. [22]. Reproduced with permission of Nature Publishing Group. (See insert for color representation of the figure.)

the integration of two devices in tandem [21,22]. The first microfluidic chip is a silicon/glass hybrid that is used to separate white blood cells and CTCs from whole blood sample using size-based deterministic lateral displacement separation technique. Then, these separated cells are transferred to the second PDMS microfluidic chip, which can align the cells using inertial focusing and sort the CTCs by magnetophoresis. A schematic of the system is shown in Figure 8.2. The system was built on a custom rig, and successful isolation of CTCs from a very populated solution was demonstrated. Although not demonstrated in this study, the identification and counting of CTCs can also be integrated using the benefits of continuous flow platforms. 
The continuous microfluidic platforms also resulted in several commercial products for disease diagnosis that are remarkable success stories in the field. One of the earliest examples of such systems is Agilent's on-chip gel capillary electrophoresis system, which is composed of a benchtop analyzer unit and single-use cartridges for DNA, RNA, and protein studies [23]. The system works with $1-5 \mu \mathrm{L}$ of samples and dramatically reduces the sample and reagent consumption. The channels are fabricated in glass and bonded to a plastic housing containing wells for sample, gel solution, and control solutions as seen in Figure 8.3. As the sample is electroosmotically run through a microfluidic channel, separation is achieved, imaging is performed using a laser-induced fluorescence unit, and the results are displayed as electropherograms. The concentration of each sample is calculated by using the calibration control standards. In addition to electrophoretic separation, a cell-based cartridge is also developed for the same platform to monitor transfection efficiency and protein expression and to study apoptosis and gene silencing mechanisms.

Another seminal example of continuous microfluidic platforms is i-STAT whole blood analysis system. This handheld unit provides a platform for measurement of blood gases, electrolytes, cardiac markers, and coagulation factors in the whole blood. The disposable cartridges require only a drop of whole blood and perform amperometric/potentiometric measurement using microfabricated electrodes fabricated on silicon chips and integrated with polymer microfluidic channels. The latest generation of i-STAT cartridges performs

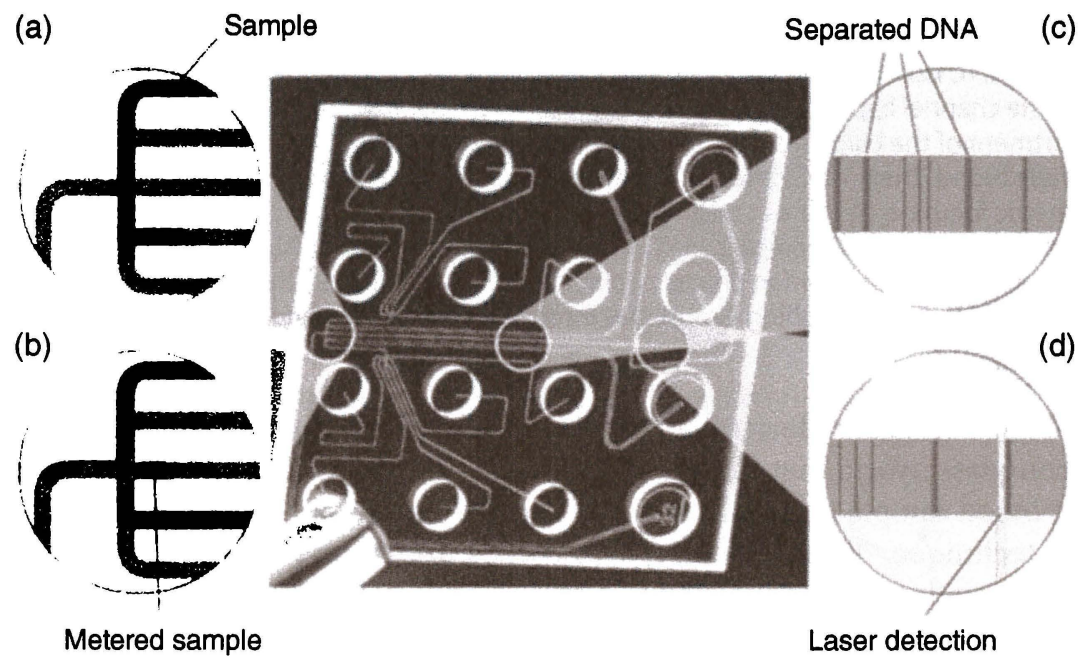

Figure 8.3 Agilent's capillary electrophoresis chip. (a) Sample is delivered to the junction area. (b) Small amount of sample is metered into the detection channel. (c) DNA is electrophoretically separated. (d) Separated DNA is fluorescently detected. Source: Mark et al. [23]. Reproduced with permission of Agilent Technologies, Inc. 
ELISA assays using on-chip washing/labeling fluids, which proves a very strong platform for the detection of biomarkers for rapid diagnosis of myocardial infarction. These systems were inspiring platforms that fueled the microfluidics research starting from its early years.

A remarkable platform for one of the latest applications of continuous fluidic systems is Ion Torrent's next-generation DNA sequencing system [24]. As opposed to the optical detection-based sequencing technologies, Ion Torrent provides a highly scalable and CMOS-based unit that provides rapid DNA sequencing. The cartridges are composed of microwells with integrated ion-sensitive field-effect transistors (ISFETs) that effectively work as micrometersize $\mathrm{pH}$ meters. Each well contains microbeads with different DNA templates. The nucleotides are flushed sequentially over the wells, and the incorporation of the nucleotide to the DNA strand in a specific well is detected as a $\mathrm{pH}$ change as schematically summarized in Figure 8.4. The system has rapidly evolved since 2010 with increased read lengths, higher number of wells, and improved read accuracies.

\subsection{Paper-Based LOC Platforms}

The intrinsic properties of paper make it a unique choice for microfluidic platforms designed for diagnosis. Paper is composed of entangled cellulose fibers forming a porous structure that makes it hydrophilic. By capillary force, paper can be used as a transport medium without any external power source. Besides, paper is ubiquitous, cheap, biodegradable, and lightweight and can be modified by chemicals; thus, it suits well for biomedical applications and diagnosis [25-30]. Paper-based platforms have existed long before the birth of microfluidics due to the availability and remarkable benefits of paper. It is only after the introduction of several LOC platforms that paper has been re-evaluated as a separate platform that gave rise to Microfluidics 2.0 [31].

In order to control the fluid flow along paper, one needs to define channels on the paper. Development of hydrophobic regions on paper with paraffin and wax to obstruct fluid flow is not a new method and is patented a century ago [32]. It is difficult to spot the earliest functional paper-based diagnostic device due to the lack of records. Yagoda's work in 1937 stands out since it demonstrated the patterning of selected areas on paper using a paraffin stamping method [33] similar to today's paper-based devices. Ring-shaped analysis spots were defined on the paper and employed for the detection of nickel and copper metal ion concentrations.

Conventional paper-based devices are categorized as dipstick and lateral flow devices. The first examples of dipstick paper detection devices were developed by a French chemist, named Jules Maunmené, in 1850 for urine analysis. In order to measure $\mathrm{pH}$ of the blood, test strips were introduced in 
(a)

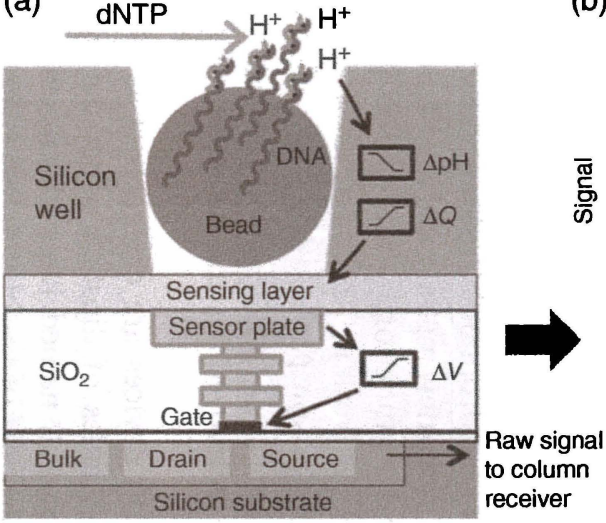

(b)

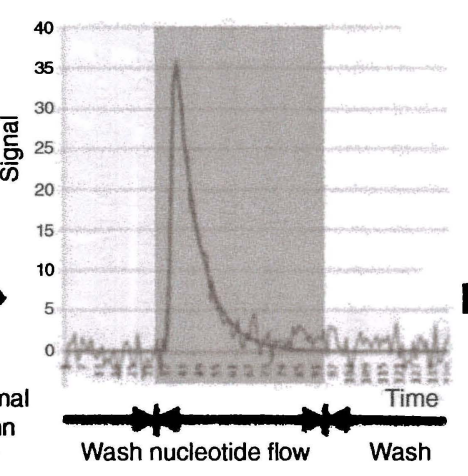

(c)

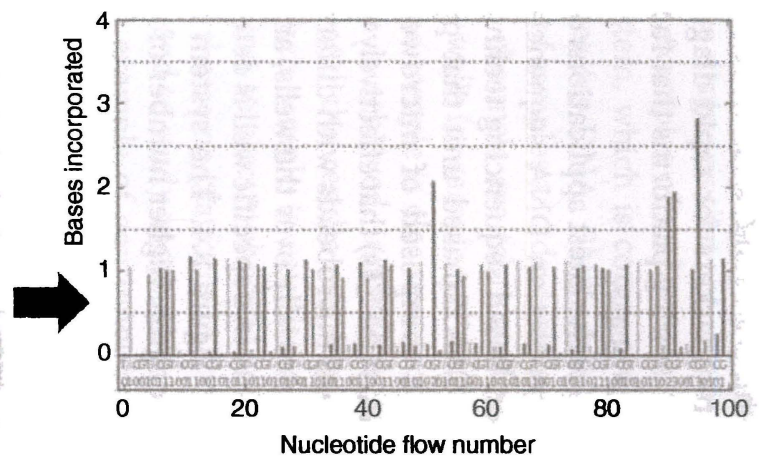

Figure 8.4 (a) Complete single-stranded DNA is cut into small pieces, and every piece is bonded on a single bead for amplification to cover all surfaces of the bead where it is placed on a small pH sensitive well. (b) Nucleotides are flowed through the chip; if there is a conjugate base in the well, then it is incorporated, releasing $\mathrm{H}^{+}$ions. Changing $\mathrm{pH}$, resulting from $\mathrm{H}^{+}$ions, is detected by $\mathrm{CMOS}$ sensor underneath the well. (c) Signals obtained from each well are encoded to obtain the DNA sequence. Source: Merriman et al. [24]. Reproduced with permission of John Wiley \& Sons. 
the 1920s and are still in the market. The widespread use of $\mathrm{pH}$ strips is due to their simplicity. By dipping the strip into the solution of interest, the $\mathrm{pH}$ of the solution (or for other assays, the analyte concentration) can be determined by a color change on the strip. The $\mathrm{pH}$ value can be determined visually using the reference indicator card. However, dipstick tests require manual sample preparation, incubation, and washing steps.

The introduction of lateral flow strips turned the simple dipstick assays into sample-in-result-out paper-based integrated devices that can perform ELISA tests. Lateral flow strips are composed of four main parts. The sample is introduced to the device from the sample inlet port. Then, the sample migrates through the second component, the conjugate pad, which includes reagents and allows for the interaction between the sample and the reagent. Moving through the conjugate pad, the sample reaches the membrane that is generally made of nitrocellulose. Across the membrane, two lines of capture antibodies were immobilized, the first one being an indicator of the presence of analyte and the second one indicating the successful transport of the sample along the membrane. Finally, the sample is collected at the absorbent pad, which is used to soak and store the leftover sample. Both sandwich and competitive assays are implemented as lateral flow assays.

Recently, paper-based platforms have gained a momentum with the advancements in the field of microfluidics [34]. Democratization of diagnostic platforms is an increasing need all across the world, which constrains the complexity of microfluidic components on a single device when cost per test is a major concern. Therefore, researchers moved to paper-based devices, and the field has experienced the resurrection of 100-year-old paper-based systems with advanced functionalities. The developments in microfabrication, printing methods, microelectronics, and microfluidics opened new avenues for paperbased platforms that allow high-scale integration with improved sensitivity and multiplexing [34].

The main enabling factor for the rebirth of paper-based platforms was the development of various low-cost fabrication techniques. In the conventional lateral flow strips, the paper channels were defined simply by cutting. However, in the second-generation paper-based platforms, the channels are defined by creating hydrophobic and hydrophilic regions across the paper (Figure 8.5). In order to pattern the hydrophilic channel region surrounded by hydrophobic barriers, there are fundamentally two approaches: physical and chemical modifications. The paper substrate can be physically modified by the impregnation of hydrophobic materials such as wax, SU-8, PDMS, or polystyrene, which not only turns the fibrous structure to hydrophobic but also blocks the pores. Another approach is to chemically modify the paper using cellulose reactive substances such as alkenyl ketene dimer (AKD). The choice of the fabrication technique is usually dominated by the cost and the minimum feature size required for the channels. Among physical modification techniques, wax 


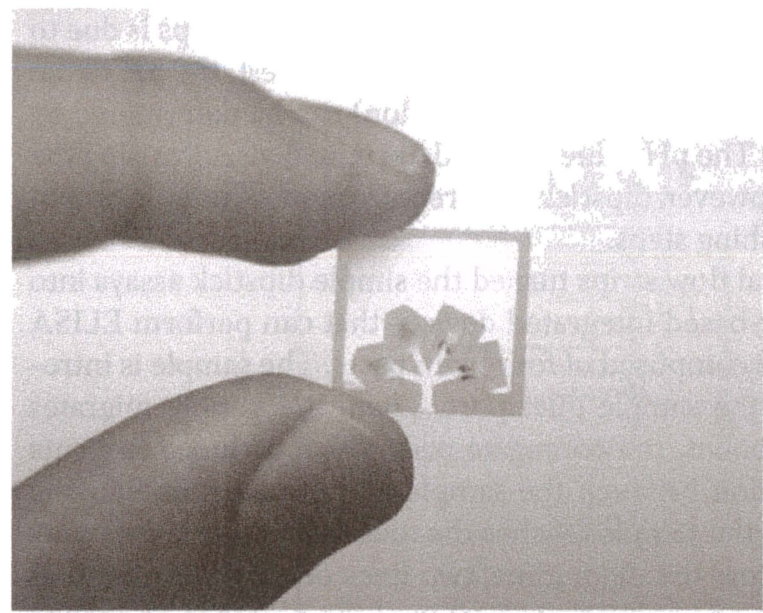

Figure 8.5 Illustration of a single-layer microfluidic paper-based device fabricated by photolithography. Source: Martinez et al. [35]. Reproduced with permission of American Chemical Society.

printing is favorable since it is cheap and practical to implement [29]. It only requires a solid wax printer and an oven to first print and then reflow the wax to be absorbed by paper, respectively. This method is mostly used when lowresolution channels suffice for the application. Similarly, PDMS dissolved in hexane or SU-8 negative photoresist [35] is also used for the hydrophobization of paper since these are commonly available materials across most microfluidics laboratories, thanks to the soft lithography process. However, compared to wax printing, SU-8 and PDMS are much costly alternatives. An additional benefit of PDMS-modified paper structures is their flexibility for applications that make use of foldable structures or 3D geometries [26]. The use of photoresist patterning on paper is especially useful when obtaining fine features on paper that are defined through photolithography.

For chemical modification of paper, AKD is the most commonly used agent to create hydrophobic regions that are defined on paper directly using an ink-jet printer [36]. This is a fairly low-cost single-step method. In comparison, some studies demonstrated that the whole paper is turned hydrophobic first, and then the hydrophilic regions are redefined by plasma treatment, employing a two-step method [37]. Among these fabrication techniques, printingbased methods are very advantageous for batch manufacturing of paper-based devices. In addition, the printing technology can also be used for printing of reagents required for the assay using a second head, which allows a fully integrated fabrication scheme that lowers the fabrication cost significantly.

Using the aforementioned techniques, several interesting paper-based devices have been demonstrated, such as detection of glucose and proteins 
(Figure 8.5). In addition to these single-layer structures, stacked multilayer paper devices were also demonstrated in more intricate geometries [38]. Similar to multilayer printed circuit boards, fluids can move across different layers through hole-like connections that are similar to vias in printed circuit boards. Such designs achieve complex fluid flow in a smaller footprint.

The most common detection methods used for paper-based platforms are unarguably colorimetric (Figure 8.6a) and electrochemical detection (Figure $8.6 \mathrm{~b}$ ). Colorimetric detection is mostly used for qualitative analysis since it merely depends on a color change based on an enzymatic reaction. One of the earliest demonstrations of colorimetric paper-based detection devices is from Martinez et al., which demonstrates the detection of BSA and glucose using a chromatography paper patterned with SU-8 photoresist. Using an artificial urine solution, detection of two analytes was demonstrated as a semiquantitative assay as seen in Figure 8.6a [39]. Similarly, well-known enzymatic colorimetric assays were implemented on paper demonstrating detection of uric acid [41], human IgG [42], and bacteria [43].

In an attempt to obtain more quantitative results, electrochemical detection has been developed on paper platforms. Dungchai et al. demonstrated detection of glucose, lactate, and uric acid by integrating electrodes on the paper defined by SU-8 patterning as shown in Figure 8.6b [40]. The low-cost

(a)

\begin{tabular}{|c|c|c|}
\hline $\begin{array}{c}\text { [glucose] } \\
(\mathrm{mM})\end{array}$ & & $\begin{array}{c}{[\mathrm{BSA}]} \\
(\mu \mathrm{M})\end{array}$ \\
\hline 0 & & 0 \\
\hline 2.5 & & 0.38 \\
\hline 5.0 & & 0.75 \\
\hline 10 & & 1.5 \\
\hline 50 & & 7.5 \\
\hline 500 & & 75 \\
\hline
\end{tabular}

$5 \mathrm{~mm}$ (b)

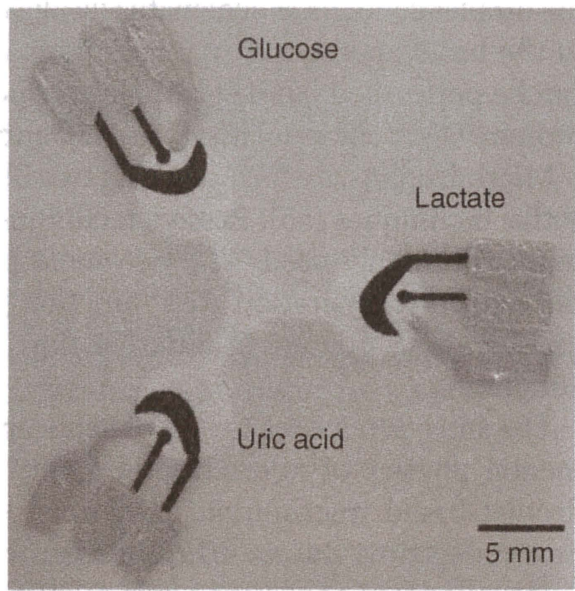

Figure 8.6 Different detection methods used in a paper-based platform. (a) Colorimetric detection of glucose and protein. Source: From Martinez et al. [39]. Adapted with permission of John Wiley \& Sons, Inc. (b) Electrochemical detection of three analytes implemented on a single device. Source: From Dungchai et al. [40]. Reprinted with permission of American Chemical Society. 
fabrication of screen-printed carbon or $\mathrm{Ag} / \mathrm{AgCl}$ electrodes was in line with the benefits of paper-based devices. Therefore, this approach has been widely adapted and opened another dimension for paper-based diagnostic devices.

Overall, paper devices are prone to variability due to environmental conditions such as temperature and humidity. Their clinical performance lags behind other LOC platforms in terms of specificity and sensitivity. In addition, they require larger sample volume due to the retention of the sample in the device during fluid transport. Still, the facts that these systems do not require off-chip components and are fairly low-cost compared to their counterparts indicate that they are attractive alternatives for affordable diagnostic applications.

\subsection{Droplet-Based LOC Platforms}

Droplet-based platforms are based on the generation and control of minute volumes of microdroplets (dispersed phase) within carrier liquids (continuous phase) inside microchannels. Individual droplets with low volumes allow very precise control over the concentration of the samples of interest as well as the reaction times. In addition, rapid and extremely repeatable generation of monodisperse droplets makes these systems suitable for high-throughput biochemical applications [44, 45]. Basic microfluidic operations that can be carried out using these platforms include transporting, splitting, and mixing of the droplet contents as schematically illustrated in Figure 8.7 [46-50]. In addition to the basic operations, manipulations that change the phase of the droplets can be performed. Some examples include chemical polymerization [51], cell/ protein/DNA encapsulation [52, 53], and particle synthesis [54].

Microdroplets are formed using two immiscible fluids using either passive or active techniques [55]. Passive techniques lead to higher throughputs and are more commonly used. The two most preferred geometries used for passive microdroplet generation are T-junction [56] and flow-focusing [57] geometries that can both operate in different flow regimes such as squeezing, dripping, and jetting.

The introduction of the $\mu$ TAS concept and the investigation of the fundamental physics of microdroplet formation led to the emergence of various droplet-based microfluidic platforms, which are advantageous for reduced processing time due to efficient micromixing inside droplets as well as performing operations (reaction, synthesis, etc.) using pico/nanoliters of liquids isolated inside droplets in a parallel way [58]. For the application of dropletbased platforms for disease detection and diagnosis, optical and electrochemical detection systems are integrated into the LOC platforms. For example, Boedicker et al. developed a system that measures antibiotic susceptibility of bacteria inside microdroplets for drug screening studies [59]. Pekin et al. demonstrated a droplet microfluidic device for highly sensitive quantitative 
(a) Droplet generation
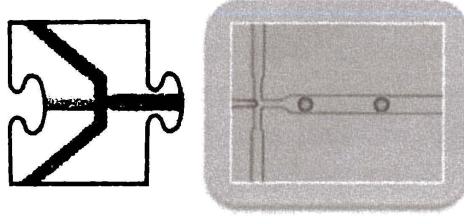

(c)

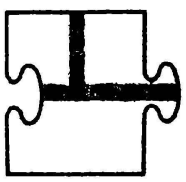

Fusion

(e) Stationary storage
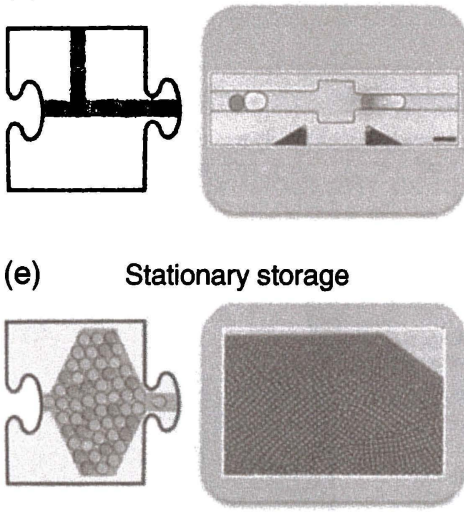

(g)

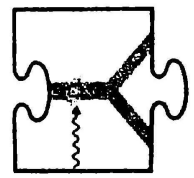

(b) Mixing and generation
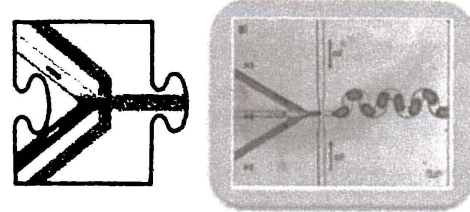

(d) Short-term incubation
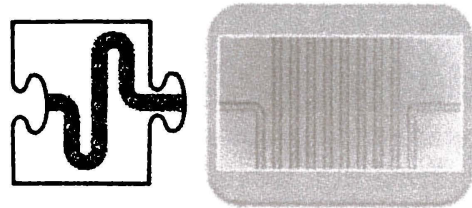

(f)

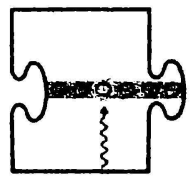

Detection

(h)

Reinjection

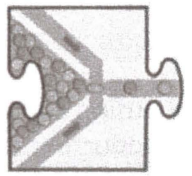

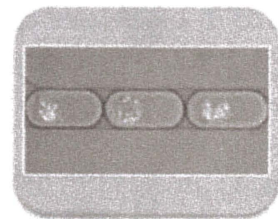

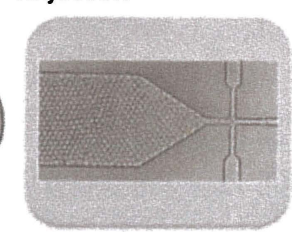

Figure 8.7 A collection of droplet unit operations: (a) droplet generation [46], (b) mixing and generation [47], (c) fusion [48], (d) incubation, (e) storage [49], (f) detection, (g) sorting, and (h) re-injection [46]. Source: From Kintses et al. [50]. Adapted with permission from Elsevier. (a and h) Source: Schaerli and Hollfelder [46]. Reproduced with permission of Royal Society of Chemistry. (b) Source: Huebner et al. [47]. Reproduced with permission of The Royal Society of Chemistry. (e) Source: Courtois et al. [49]. Adapted with permission of John Wiley Sons, Inc. (See insert for color representation of the figure.)

detection of mutated DNA within complex DNA mixtures as shown in Figure 8.8 [60]. The system performs digital PCR in millions of droplets with picoliter sample volumes obtaining the required sensitivity and specificity to detect rare tumoral DNA in diverse samples. Such an application is very critical for diagnosis of genetic disorders.

Also, droplets can be used for the functional characterization of cell libraries at high throughput by capturing single cells inside microdroplets. Debs et al. developed a platform containing modules for the generation, incubation, 
(a)

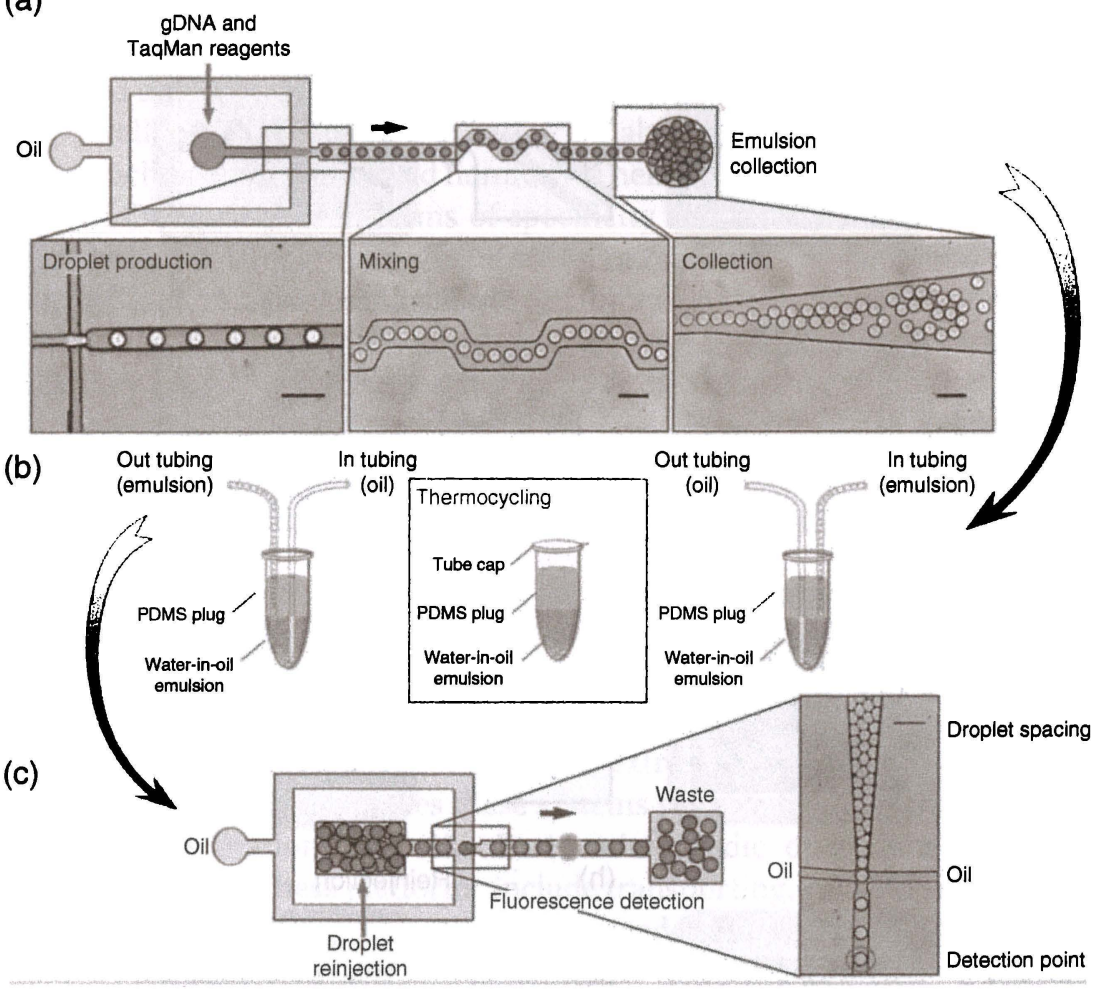

Figure 8.8 Detection of mutated DNA using digital PCR in picoliter microdroplets. (a) An overview of the system showing the production, mixing, and collection of droplets containing DNA, PCR reagents, and TaqMan probes. (b) Thermocycling and amplification of the emulsion. (c) Reinjection of droplets and analysis of the fluorescence signal of individual droplets. Source: Pekin et al. [60]. Reproduced with permission of Royal Society of Chemistry.

fusion, and sorting of droplets for hybridoma screening [61]. Since individual cells are isolated inside single droplets, molecules secreted from a single cell can be attained in relatively high concentrations.

Using droplet-based platforms allows detection of diseases at extreme sensitivity and specificity. For example, Juul et al. presented a novel system for the detection of single parasites that cause malaria [62] from a drop of whole blood and saliva samples using in-droplet rolling circle DNA amplification, which does not require thermal cycling. Marcali et al. developed a droplet-based impedimetric hemagglutination detection system that can potentially be used for any agglutination-based study inside microdroplets. The system requires no sample preparation and achieves side injection of blood sample into serum droplets containing agglutinins that are proceeded by electrical detection of agglutination of erythrocytes inside a nanoliter-sized droplet [63]. 
Despite the high sensitivity obtained using droplet platforms, their commercialization stayed limited mainly due to the relatively complicated system design and the need for several off-chip components. Bio-Rad and RainDance Technologies are two leading companies that produce droplet-based digital PCR devices. More recently, Sphere Fluidics has commercialized a droplet microfluidic system for the analysis and detection of cellular genomic screening.

\subsection{Digital Microfluidic-Based LOC Platforms}

DMF-based platforms are based on electrowetting-on-dielectric (EWOD) that allows precise and effective control of droplets (for transporting, sorting, merging, etc.) without any need for microchannels [64]. On DMF platforms, droplets are manipulated along virtual channels that are created by sequential activation and deactivation of electrodes that are laid underneath the fluid layer. This is unlike the droplet microfluidic devices explained in the previous section where the liquids are carried inside microchannels and are driven by pumping. This capability frees the DMF systems from all pump and valve units, resulting in compact, electrically controlled LOC platforms as schematically shown in Figure 8.9 [65]. Thanks to the droplet-based operation, DMF systems also benefit from low reagent volume, efficient reagent mixing, and lower reaction times.

EWOD is a phenomenon where one can modify the interfacial tension of polarizable and/or conductive droplets by applying an electric field to hydrophobic, insulated electrodes [66]. This change in the interfacial tension results

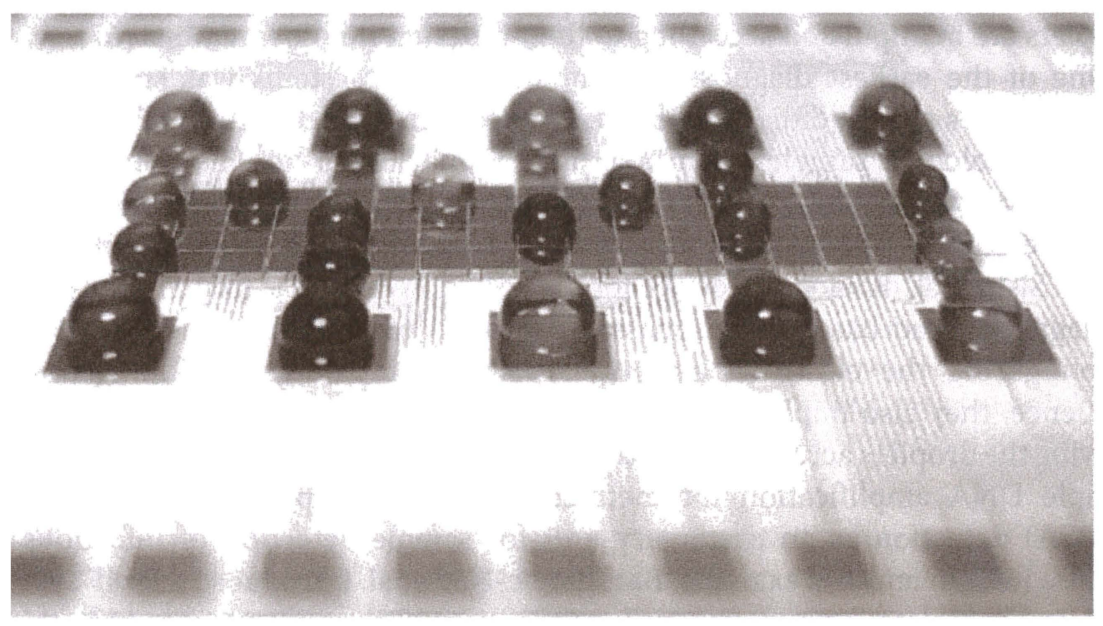

Figure 8.9 A sample digital microfluidic platform system that is capable of working with multiple droplets without requiring any channels, pumps, or valves. Source: Jebrail and Wheeler [65]. Reproduced with permission of Elsevier. 
in a change in the wettability, therefore contact angle, of the droplet on the surface. This relation is described by the Lippmann-Young equation as follows:

$$
\cos \theta(V)=\frac{C V^{2}}{2 \gamma}+\cos \theta_{0}
$$

where $\theta_{\mathrm{o}}$ and $\theta$ are the contact angles between the droplet and the surface before and after the voltage application, respectively, $C$ is the capacitance per unit area of the dielectric and the hydrophobic layers, and $\gamma$ is the interfacial tension between the droplet and the surrounding medium. By turning the voltage "on" and "off", an interfacial tension gradient is formed. This allows the individual droplets to move across the electrodes. Usually, AC actuation is preferred over DC to avoid insulator charging. Based on the application, different media can be used for droplet manipulation. In order to tackle with the evaporation problem, the system can be run in an oil bath.

DMF platforms have two main electrode configurations: single-plane and two-planes (Figure 8.10). In the single-plane configuration, the actuation and ground electrodes are on the bottom surface leaving the fluids of interest open to air (open system). Alternatively, the fluids can be encapsulated by passive covers without compromising system performance. In the two-planes configuration, the ground electrode and actuation electrodes are separated by a spacer and are located on the top and bottom surfaces, respectively (close system). The latter configuration is often used for automated manipulation of droplets in biomedical and chemical fields. The fabrication processes of single-plane and two-plane configurations are similar. Both configurations consist of a substrate, actuation electrodes, and dielectric surface, which are coated with hydrophobic layer. The device can be partitioned into virtual channels in which droplets are manipulated according to their different fluidic functions [67].

One of the earliest diagnostic examples of DMF systems was shown by Richard Fair's group [68]. They showed the colorimetric detection of glucose from physiological samples such as serum, plasma, and urine. The system also demonstrated that multiple solutions can be studied in parallel with a monolithic device. Another critical feature of DMF platforms is their reconfigurability. Sista et al. developed a device for the detection of cardiac troponin-I using a magnetic bead-based immunoassay protocol [69]. On the same system, reconfiguring the electrode actuation, that is, droplet generation/motion sequence, they also implemented a real-time DNA amplification protocol by moving the droplets across temperature zones. The system was used to perform sample DNA amplification for methicillin-resistant staphylococcus aureus (MRSA) detection as an exemplary unit for sample detection of infectious disease [69]. In another example, Barbulovic-Nad et al. introduced a DMF system for complete mammalian cell culture including cell seeding, growth, detachment, and reseeding steps [70]. The system proved that adherent cells can be cultured using this novel platform, which was verified by proliferation 
(a) Ground electrode

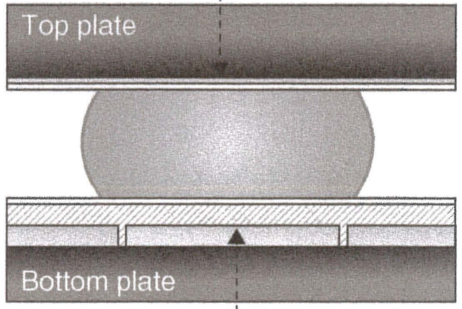

Actuation electrode (b)

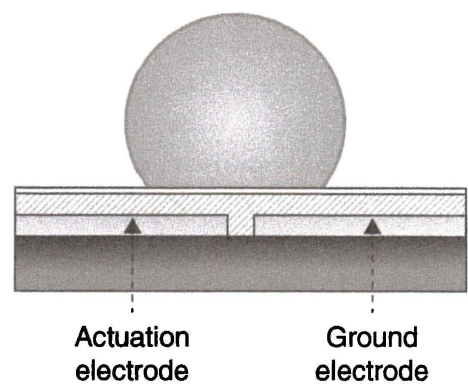

(c)

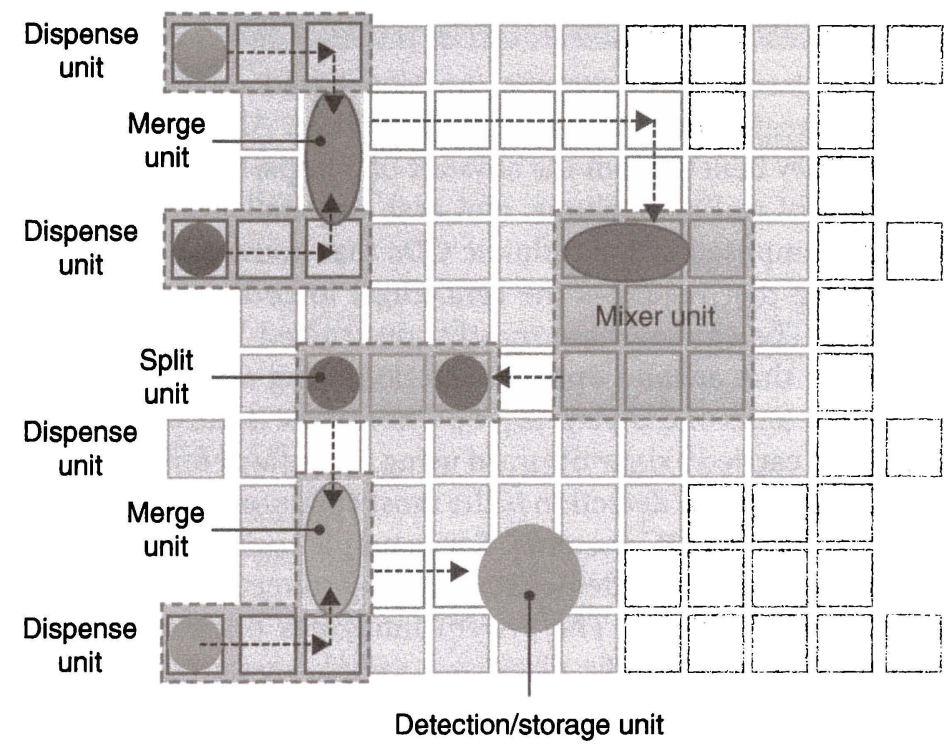

Figure 8.10 Digital microfluidic platforms: (a) Close system and (b) open system. (c) 2D schematic illustration of digital microfluidic chip illustrating dispensing, merging, splitting, and mixing units. Source: Malic et al. [67]. Reproduced with permission of Royal Society of Chemistry. (See insert for color representation of the figure.)

studies on two cell lines. As a more recent example on cellular studies of DMFs, $\mathrm{Ng}$ et al. demonstrated immunocytochemistry in single cells [71]. The assay was performed on adherent cells that are fixed on the top plate. The system was optimized so that shear stress is kept low during medium exchange over 
the cells while achieving precise and automated fluid delivery. This system can be further extended as a universal platform to study single-cell protein expressions or cell signaling pathways.

A few companies have launched products based on DMFs for biological and chemical detection [72]. It is especially noteworthy that due to the ease of platform construction, automation, and low-cost fabrication, these platforms possess the possibilities to be employed for the detection of a wide variety of biomarkers (DNA, protein, cell, etc.) for disease detection [73]. For example, Advanced Liquid Logic Inc., acquired by Illumina in 2013, developed a benchtop system that can perform DNA, RNA, and protein analyses, proving its high potential as a multi-disease detection platform. Sci-bots is a recent spin-off from Prof. Aaron Wheeler's group aiming for a customizable DMF platform for biochemical diagnostics, cell-based assays, and chemical synthesis.

\subsection{CD-Based LOC Platforms}

Compact disc (CD)-based LOC systems function based on the rotational motion of $C D$ that creates an outward centrifugal force. Also known as labon-a-disc systems, they benefit from the absence of pumps, bubble-free liquid motion, elimination of residual volume, and ease of parallelization [74, 75]. These systems are composed of microfluidic CDs that house the fluids and a $\mathrm{CD}$ player-like device that regulates the centrifugal force by controlling the rotation rate $(\mathrm{rpm})$. The channels are mostly constructed using transparent plastics (Figure 8.11) that enables optical detection using a hardware that is similar to the readout units of CD players [76]. The sample is introduced to the center of the $\mathrm{CD}$ and carried axially outward using centrifugal force.

Not too surprisingly, optical detection is the most commonly used detection mechanism for CD-based LOC systems. For example, using a colorimetric detection system, Nwankire et al. demonstrated a 5-parameter liver panel test that operates with whole blood. The system integrates plasma separation, which is trivial for $C D$ microfluidic platforms, sample metering, aliquoting, and parallelized detection, which completes the assay in 20 min using less reagent compared to conventional systems [77]. Similarly, fluorescence quantum dots are employed for ultrasensitive detection of botulinum neurotoxin from whole blood, serum, and saliva samples on a CD-based platform [78]. This work presents the implementation of all immunoassay steps on the system, which can readily be adapted for other biomarkers as well. These advanced optical detection systems have their roots in one of the pioneering works in CD microfluidic platforms used for label-free detection of biomolecules [79]. In this study, ligands are immobilized on a CD for immunoassay capturing of molecules. Using the optical setup within the optical disc drive, the error rate of the $C D$ was recorded, and a high correlation was found between the 
(a)

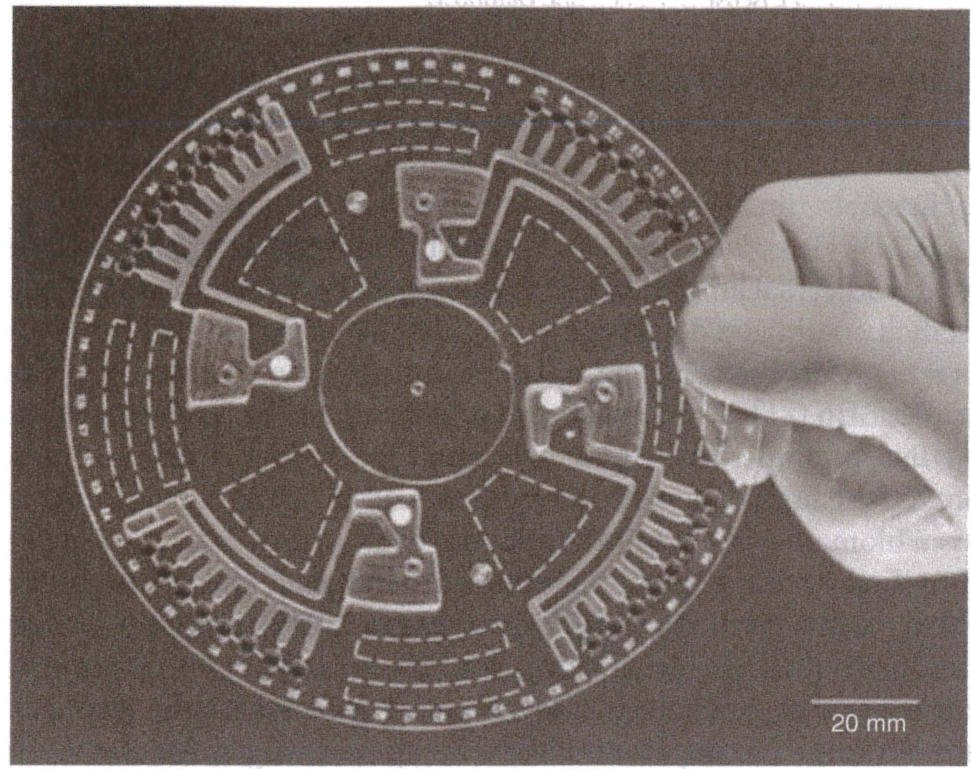

(b)
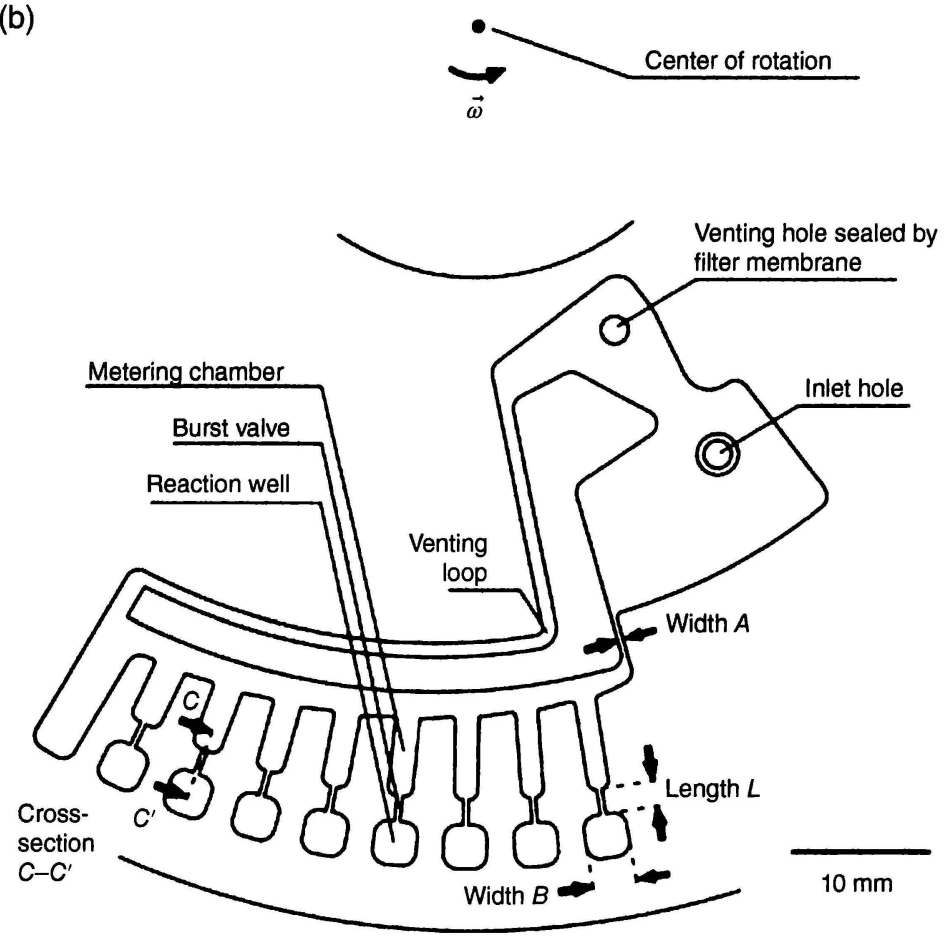

Figure 8.11 (a) Photograph of a CD microfluidic cartridge unit, reaction wells are filled with dye solution. (b) Schematic of the microfluidic design showing some critical components such as inlet, metering chambers, burst valves, and reaction wells. Source: Focke et al. [76]. Reproduced with permission of Royal Society of Chemistry. (See insert for color representation of the figure.) 
biomolecular concentration and the error rate. Centrifugal microfluidic platforms can be used in conjunction with other microfluidic platforms as well. For instance, in a recent study, a CD microfluidic unit is combined with an immunochromatographic strip assay for the very selective detection of influenza $\mathrm{A}(\mathrm{H} 1 \mathrm{~N} 1)$ virus detection [80]. The system uses reverse transcriptase loop-mediated isothermal amplification and proves to be a dexterous platform for high specificity pathogen detection.

In an interesting work, electrochemical detection was implemented on a CD microfluidic platform [81]. Electrical slip rings were used to make contact with the spinning disc for amperometric electrochemical detection. The presented system improved the limit of detection for the measurement of $\mathrm{C}$-reactive protein, which is a biomarker for cardiovascular diseases.

Optical disc drive technology that has been around since the 1980s can be easily modified for the formation of CD-based LOC platforms. Optical disc drives contain high-quality and fully integrated laser diodes, photodetectors, lenses, and motors that facilitate the development process for the analyzer. Then, what is left is the development of the CD-type cartridges, which inherit the fabrication methodologies of CDs from the music industry. Therefore, their commercialization was relatively easier compared to the other platforms that require several off-chip components. One of the most well-known centrifugal-based microfluidic companies is Abaxis, which was founded in 1989. Abaxis' Piccolo Xpress system provides a very wide variety of panel assays such as metabolic panel, liver panel, general chemistry, electrolyte panel, and lipid panel. Gyros is another leading company offering CD-based LOC systems solutions targeting several applications ranging from immunogenicity, toxicokinetics, biomarker detection to pharmacodynamics, and impurity testing for therapeutic studies.

\subsection{Wearable LOC Platforms}

Wearable LOC platforms have been receiving growing interest from academia due to their capacity for reliable health monitoring of wearers. They enable us to obtain information regarding an individual's state of health in a real-time, continuous, and noninvasive manner [82]. Thus, what is aimed is an increase in the quality of life as well as reduction in medical costs associated with diagnostics and therapy $[83,84]$. The detection principle is mostly based on electrochemical sensing that provides vital information by measuring physiological analytes from sweat, saliva, or tear. In particular, glucose and lactate are targeted for real-time disease monitoring [85]. Whole blood, serum, or urea, which are mostly used by other LOC platforms, provide higher concentrations and more precise measurements. On the flip side, it is very challenging to make continuous analysis using these fluids. Sweat, saliva, and tear are relatively easy 
to continuously sample and therefore can be potentially used for continuous, noninvasive monitoring of analytes using wearable platforms. For example, concentrations of the electrolytes ( $\mathrm{pH}$, sodium, calcium) and metabolites (glucose, lactate) in sweat provide vital health information $[84,86]$. Moreover, thanks to their printed embodiments, these platforms do not have to employ optical or microelectromechanical components, leading to inexpensive and robust sensing systems [87-89].

Saliva known as a very complex biofluid can be employed to monitor hormonal, nutritional, and metabolic parameters [90]. The first LOC platforms aimed at monitoring plaque $\mathrm{pH}$ (for studying plaque acidogenicity) and fluoride (for studying fluoride dentifrice efficiency) in saliva [91]. Recently, amperometric saliva LOC platform with wireless transmission feature is fabricated, which measures lactate concentration continuously and noninvasively [92]. In another study, antimicrobial peptides are transferred on a graphene-modified silk substrate for continuous bacterial monitoring [93].

Human sweat can also be used to obtain very important information. For example, sodium for electrolyte imbalance, lactate for cystic fibrosis, ammonium for stress, and calcium for bone mineral loss can be monitored [84]. Sweat can also be employed to measure toxicant [94] and drug-of-abuse concentrations [84]. Sweat-based wearable LOC platforms can be categorized into two types: fabric/flexible plastic-based and epidermal-based LOC platforms. In fabric-based platforms, there is a stable contact between skin and sensor, which allows real-time and continuous measurement of targeted biomarkers in the sweat. Flexible materials used within the platforms allow selective and simultaneous measurement of glucose, lactate, sodium, and potassium ions, as well as skin temperature [82]. Studies for monitoring sodium [95], potassium [96], and chloride levels [97] are also available in the literature. Epidermalbased platforms are mostly tattoo-based LOC platforms where electrodes are printed on temporary tattoos that can have intimate contact with the body for electrochemical sensing [98]. These tattoos are biocompatible, single use, highly resilient to mechanical deformation, and aesthetic, and they do not interfere with the daily routine of the user $[84,86,98]$. The first tattoo-based LOC platform was reported in 2012 [98]. Here, a Ag/ $\mathrm{AgCl}$ ink is screen printed on a temporary tattoo as an electrode.

Tear contains peptide, electrolytes, lipids, metabolites, ocular epithelial cells, and blood. As such, it is an attractive body fluid for health monitoring. Also, glucose concentrations in blood and tear are shown to correlate well [99]. However, tear is prone to rapid evaporation in vitro, limiting its use for POC platforms. The initial studies fabricated bare gold or platinum electrodes on flexible substrates to form strip sensors using photolithography [100]. These studies showed monitoring of transcutaneous oxygen and glucose. However, they were designed such that they caused reflex tear and did not have integrated electronics [84]. The Parviz group at the University of Washington 
developed a contact lens-based sensor integrated with wireless electronics $[101,102]$. The sensor employs glucose oxidase enzyme for continuous glucose monitoring. Google (Alphabet Incorporation) is further developing this sensor system. GlucoWatch is a commercial product that continuously monitors glucose level from interstitial fluid [103], which has been used to measure metabolic diseases, organ failure, drug efficiency, and glucose concentration. The consumers have reported skin irritation, causing the withdrawal of the product from the market. More studies are required to fully utilize interstitial fluid for wearable platforms.

\subsection{Conclusion and Outlook}

The idea of forming miniaturized integrated systems for biomedical applications has gained popularity within the past decades. Especially, the efforts to combine numerous laboratory tasks on a single chip, that is, LOC, for more effective medical research are noteworthy. This chapter summarized most common LOC platforms that are of use for disease detection and diagnosis. Seminal studies as well as the state of the art in literature were provided. Additionally, successful commercial examples in the market were discussed.

The LOC platforms are promising alternatives to the bulky, lab-based diagnostic equipment. So far, these systems have been mostly adapted in the developed world for rapid diagnostic needs at emergency settings or for advanced biochemical analysis. At the developing world, the driving force for the use of LOC diagnostic platforms was their low cost and simplicity. In spite of the diversity of LOC platforms and their ability to tap into different markets, the number of commercial technologies that made it to the market is still limited considering the wide variety of applications demonstrated in academic publications mainly due to the fact that the engineering of these platforms requires multidisciplinary teams and considerable budget to allow the technologies to move beyond clinical trials successfully. In order to compete with the status quo, LOC platforms have to demonstrate either advanced functionalities or significant reduction in cost. What is so motivating for the researchers in this field is the potential of these technologies to give birth to niche applications.

The twenty-first century is witnessing a shift in the healthcare services. With the increasing awareness of individuals on personal health and healthcare technologies that allow continuous monitoring, our conception of diagnostics will evolve radically. The increasing interest on personalized medicine and telemedicine are some of the indicators of this upcoming transition. Real-time investigation of the health status of a person without interfering with daily activities holds great promise for early detection of a wide variety of abnormalities. This early detection, and therefore early medical intervention, has the potential to dramatically decrease the burden of life-threatening diseases as 
aimed by wearable LOC platforms. In the near future, rather than using textile or tattoo as a substrate, these platforms can be implanted into the body for continuous monitoring of physicochemical conditions.

Since microfluidics and LOC systems were first introduced in the 1990s, we have seen studies focusing on investigating individual cells in order to understand the building blocks of tissues, organs, and organisms. More recently, a collective approach has gained traction that aims to study the interaction of cells and mimic the functionalities of organs on microfluidic platforms. These systems are called organ-on-a-chip platforms. The ultimate aim of these studies is to create a human-on-a-chip by constructing each organ on the same platform. The human-on-a-chip will simulate the activities of individual organs as well as their collective behaviors. This will revolutionize the position of LOC platforms against conventional systems and the methods used for disease diagnosis as well as personalized treatment.

\section{References}

1 Castillo-León, J.; Svendsen, W. E. Lab-on-a-Chip Devices and Micro-Total Analysis Systems: A Practical Guide; Springer, Cham, 2014.

2 Terry, S. C.; Jerman, J. H.; Angell, J. B. IEEE Transactions on Electron Devices 1979, 26, 1880-1886.

3 Bassous, E.; Taub, H.; Kuhn, L. Applied Physics Letters 1977, 31, 135-137.

4 Manz, A.; Graber, N.; Widmer, H. Á. Sensors and Actuators B: Chemical 1990, $1,244-248$.

5 Duffy, D. C.; McDonald, J. C.; Schueller, O. J.; Whitesides, G. M. Analytical Chemistry 1998, 70, 4974-4984.

6 Peeling, R. W.; Holmes, K. K.; Mabey, D.; Ronald, A. Sexually Transmitted Infections 2006, 82, v1-v6.

7 Peeling, R.; Mabey, D. Clinical Microbiology and Infection 2010, 16, 1062-1069.

8 Nahavandi, S.; Baratchi, S.; Soffe, R.; Tang, S.-Y.; Nahavandi, S.; Mitchell, A.; Khoshmanesh, K. Lab on a Chip 2014, 14, 1496-1514.

9 Isiksacan, Z.; Erel, O.; Elbuken, C. Lab on a Chip 2016, 24, 4682-4690.

10 Laser, D. J.; Santiago, J. G. Journal of Micromechanics and Microengineering 2004, 14, R35-R64.

11 Abhari, F.; Jaafar, H.; Yunus, N. A. M. International Journal of Electrochemical Science 2012, 7, 9765-9780.

12 Au, A. K.; Lai, H.; Utela, B. R.; Folch, A. Micromachines 2011, 2, 179-220.

13 Oh, K. W.; Ahn, C. H. Journal of Micromechanics and Microengineering 2006, 16, R13-R39.

14 Nguyen, N.-T.; Wu, Z. Journal of Micromechanics and Microengineering 2004, 15, R1-R16. 
15 Burns, M. A.; Johnson, B. N.; Brahmasandra, S. N.; Handique, K.; Webster, J. R.; Krishnan, M.; Sammarco, T. S.; Man, P. M.; Jones, D.; Heldsinger, D. Science 1998, 282, 484-487.

16 Unger, M. A.; Chou, H.-P.; Thorsen, T.; Scherer, A.; Quake, S. R. Science 2000, 288, 113-116.

17 Hong, J. W.; Studer, V;; Hang, G.; Anderson, W. F.; Quake, S. R. Nature Biotechnology 2004, 22, 435-439.

18 Fu, A. Y.; Chou, H.-P.; Spence, C.; Arnold, F. H.; Quake, S. R. Analytical Chemistry 2002, 74, 2451-2457.

19 Thorsen, T.; Maerkl, S. J.; Quake, S. R. Science 2002, 298, 580-584.

20 Shields, IV, C. W.; Reyes, C. D.; López, G. P. Lab on a Chip 2015, 15, 1230-1249.

21 Ozkumur, E.; Shah, A. M.; Ciciliano, J. C.; Emmink, B. L.; Miyamoto, D. T.; Brachtel, E.; Yu, M.; Chen, P.-I.; Morgan, B.; Trautwein, J. Science Translational Medicine 2013, 5, 179ra47.

22 Karabacak, N. M.; Spuhler, P. S.; Fachin, F.; Lim, E. J.; Pai, V.; Ozkumur, E.; Martel, J. M.; Kojic, N.; Smith, K.; Chen, P.-I. Nature Protocols 2014, 9, 694-710.

23 Mark, D.; Haeberle, S.; Roth, G.; von Stetten, F.; Zengerle, R. Chemical Society Reviews 2010, 39, 1153-1182.

24 Merriman, B.; Torrent, I.; Rothberg, J. M.; Team, D. Electrophoresis 2012, 33, 3397-3417.

25 Cate, D. M.; Adkins, J. A.; Mettakoonpitak, J.; Henry, C. S. Analytical Chemistry 2014, 87, 19-41.

26 Bruzewicz, D. A.; Reches, M.; Whitesides, G. M. Analytical Chemistry 2008, 80, 3387-3392.

27 Carrilho, E.; Martinez, A. W.; Whitesides, G. M. Analytical Chemistry 2009, 81, 7091-7095.

28 Hu, J.; Wang, S.; Wang, L.; Li, F.; Pingguan-Murphy, B.; Lu, T. J.; Xu, F. Biosensors and Bioelectronics 2014, 54, 585-597.

29 Lu, Y.; Shi, W.; Jiang, L.; Qin, J.; Lin, B. Electrophoresis 2009, 30, 1497-1500.

30 Wang, J.; Monton, M. R. N.; Zhang, X.; Filipe, C. D.; Pelton, R.; Brennan, J. D. Lab on a Chip 2014, 14, 691-695.

31 Osborn, J. L.; Lutz, B.; Fu, E.; Kauffman, P.; Stevens, D. Y.; Yager, P. Lab on a Chip 2010, 10, 2659-2665.

32 Dieterich, K. Device for grinding broken paper, US Patent, 730617 A, 1902.

33 Yagoda, H. Industrial and Engineering Chemistry, Analytical Edition 1937, 9, 79-82.

34 López-Marzo, A. M.; Merkoçi, A. Lab on a Chip 2016, 16, 3150-3176.

35 Martinez, A. W.; Phillips, S. T.; Whitesides, G. M.; Carrilho, E. Analytical Chemistry 2010, 82, 3-10.

36 Li, X.; Tian, J.; Garnier, G.; Shen, W. Colloids and Surfaces B: Biointerfaces 2010, 76, 564-570. 
37 Li, X.; Tian, J.; Nguyen, T.; Shen, W. Analytical Chemistry 2008, 80, 9131-9134.

38 Martinez, A. W.; Phillips, S. T.; Whitesides, G. M. Proceedings of the National Academy of Sciences 2008, 105, 19606-19611.

39 Martinez, A. W.; Phillips, S. T.; Butte, M. J.; Whitesides, G. M. Angewandte Chemie International Edition 2007, 46, 1318-1320.

40 Dungchai, W.; Chailapakul, O.; Henry, C. S. Analytical Chemistry 2009, 81, 5821-5826.

41 Li, X.; Tian, J.; Shen, W. Analytical and Bioanalytical Chemistry 2010, 396, 495-501.

42 Abe, K.; Kotera, K.; Suzuki, K.; Citterio, D. Analytical and Bioanalytical Chemistry 2010, 398, 885-893.

43 Li, C.-Z.; Vandenberg, K.; Prabhulkar, S.; Zhu, X.; Schneper, L.; Methee, K.; Rosser, C. J.; Almeide, E. Biosensors and Bioelectronics 2011, 26, 4342-4348.

44 Teh, S.-Y.; Lin, R.; Hung, L.-H.; Lee, A. P. Lab on a Chip 2008, 8, 198-220.

45 Foudeh, A. M.; Didar, T. F.; Veres, T.; Tabrizian, M. Lab on a Chip 2012, 12, 3249-3266.

46 Schaerli, Y.; Hollfelder, F. Molecular Biosystems 2009, 5, 1392-1404.

47 Huebner, A.; Sharma, S.; Srisa-Art, M.; Hollfelder, F.; Edel, J. B. Lab on a Chip 2008, 8, 1244-1254.

48 Brouzes, E.; Medkova, M.; Savenelli, N.; Marran, D.; Twardowski, M.; Hutchison, J. B.; Rothberg, J. M.; Link, D. R.; Perrimon, N.; Samuels, M. L. Proceedings of the National Academy of Sciences of the United States of America 2009, 106, 14195-14200.

49 Courtois, F.; Olguin, L. F.; Whyte, G.; Bratton, D.; Huck, W. T.; Abell, C.; Hollfelder, F. ChemBioChem 2008, 9, 439-446.

50 Kintses, B.; van Vliet, L. D.; Devenish, S. R.; Hollfelder, F. Current Opinion in Chemical Biology 2010, 14, 548-555.

51 De Geest, B. G.; Urbanski, J. P.; Thorsen, T.; Demeester, J.; De Smedt, S. C. Langmuir 2005, 21, 10275-10279.

52 He, M.; Sun, C.; Chiu, D. T. Analytical Chemistry 2004, 76, 1222-1227.

53 He, M.; Edgar, J. S.; Jeffries, G. D.; Lorenz, R. M.; Shelby, J. P.; Chiu, D. T. Analytical Chemistry 2005, 77, 1539-1544.

54 Hung, L.-H.; Choi, K. M.; Tseng, W.-Y.; Tan, Y.-C.; Shea, K. J.; Lee, A. P. Lab on a Chip 2006, 6, 174-178.

55 Zhu, P.; Wang, L. Lab on a Chip 2017, 17, 34-75.

56 Thorsen, T.; Roberts, R. W.; Arnold, F. H.; Quake, S. R. Physical Review Letters 2001, 86, 4163.

57 Ganán-Calvo, A. M.; Gordillo, J. M. Physical Review Letters 2001, 87, 274501.

58 Shestopalov, I.; Tice, J. D.; Ismagilov, R. F. Lab on a Chip 2004, 4, 316-321.

59 Boedicker, J. Q.; Li, L.; Kline, T. R.; Ismagilov, R. F. Lab on a Chip 2008, 8, 1265-1272. 
60 Pekin, D.; Skhiri, Y.; Baret, J.-C.; Le Corre, D.; Mazutis, L.; Salem, C. B.; Millot, F.; El Harrak, A.; Hutchison, J. B.; Larson, J. W. Lab on a Chip 2011, 11, 2156-2166.

61 El Debs, B.; Utharala, R.; Balyasnikova, I. V.; Griffiths, A. D.; Merten, C. A. Proceedings of the National Academy of Sciences of the United States of America 2012, 109, 11570-11575.

62 Juul, S.; Nielsen, C. J.; Labouriau, R.; Roy, A.; Tesauro, C.; Jensen, P. W.; Harmsen, C.; Kristoffersen, E. L.; Chiu, Y.-L.; Frøhlich, R. ACS Nano 2012, 6, 10676-10683.

63 Marcali, M.; Elbuken, C. Lab on a Chip 2016, 16, 2494-2503.

64 Lee, J.; Moon, H.; Fowler, J.; Schoellhammer, T.; Kim, C.-J. Sensors and Actuators A: Physical 2002, 95, 259-268.

65 Jebrail, M. J.; Wheeler, A. R. Current Opinion in Chemical Biology 2010, 14, 574-581.

66 Fair, R. B. Microfluidics and Nanofluidics 2007, 3, 245-281.

67 Malic, L.; Brassard, D.; Veres, T.; Tabrizian, M. Lab on a Chip 2010, 10, $418-431$.

68 Srinivasan, V.; Pamula, V. K.; Fair, R. B. Lab on a Chip 2004, 4, 310-315.

69 Sista, R.; Hua, Z.; Thwar, P.; Sudarsan, A.; Srinivasan, V.; Eckhardt, A.; Pollack, M.; Pamula, V. Lab on a Chip 2008, 8, 2091-2104.

70 Barbulovic-Nad, I.; Au, S. H.; Wheeler, A. R. Lab on a Chip 2010, 10, 1536-1542.

$71 \mathrm{Ng}, \mathrm{A}$. H.; Chamberlain, M. D.; Situ, H.; Lee, V.; Wheeler, A. R. Nature Communications 2015, 6, 1.

72 Volpatti, L. R.; Yetisen, A. K. Trends in Biotechnology 2014, 32, 347-350.

73 Shen, H.-H.; Fan, S.-K.; Kim, C.-J.; Yao, D.-J. Microfluidics and Nanofluidics 2014, 16, 965-987.

74 Burger, R.; Kirby, D.; Glynn, M.; Nwankire, C.; O’Sullivan, M.; Siegrist, J.; Kinahan, D.; Aguirre, G.; Kijanka, G.; Gorkin, R. A. Current Opinion in Chemical Biology 2012, 16, 409-414.

75 Tang, M.; Wang, G.; Kong, S.-K.; Ho, H.-P. Micromachines 2016, 7, 26.

76 Focke, M.; Stumpf, F.; Faltin, B.; Reith, P.; Bamarni, D.; Wadle, S.; Müller, C.; Reinecke, H.; Schrenzel, J.; Francois, P. Lab on a Chip 2010, 10, 2519-2526.

77 Nwankire, C. E.; Czugala, M.; Burger, R.; Fraser, K. J.; Glennon, T.; Onwuliri, B. E.; Nduaguibe, I. E.; Diamond, D.; Ducrée, J. Biosensors and Bioelectronics 2014, 56, 352-358.

78 Koh, C.-Y.; Schaff, U. Y.; Piccini, M. E.; Stanker, L. H.; Cheng, L. W.; Ravichandran, E.; Singh, B.-R.; Sommer, G. J.; Singh, A. K. Analytical Chemistry 2015, 87, 922-928.

79 La Clair, J. J.; Burkart, M. D. Organic and Biomolecular Chemistry 2003, 1, 3244-3249.

80 Jung, J.; Park, B.; Oh, S.; Choi, G.; Seo, T. Lab on a Chip 2015, 15, 718-725.

81 Kim, T.-H.; Abi-Samra, K.; Sunkara, V.; Park, D.-K.; Amasia, M.; Kim, N.; Kim, J.; Kim, H.; Madou, M.; Cho, Y.-K. Lab on a Chip 2013, 13, 3747-3754. 
82 Gao, W.; Emaminejad, S.; Nyein, H. Y. Y.; Challa, S.; Chen, K.; Peck, A.; Fahad, H. M.; Ota, H.; Shiraki, H.; Kiriya, D. Nature 2016, 529, 509-514.

83 Appelboom, G.; Camacho, E.; Abraham, M. E.; Bruce, S. S.; Dumont, E. L.; Zacharia, B. E.; D’Amico, R.; Slomian, J.; Reginster, J. Y.; Bruyère, O. Archives of Public Health 2014, 72(1), 28.

84 Bandodkar, A. J.; Wang, J. Trends in Biotechnology 2014, 32, 363-371.

85 Gowers, S. A.; Curto, V. F.; Seneci, C. A.; Wang, C.; Anastasova, S.; Vadgama, P.; Yang, G.-Z.; Boutelle, M. G. Analytical Chemistry 2015, 87, 7763-7770.

86 Bandodkar, A. J.; Jia, W.; Wang, J. Electroanalysis 2015, 27, 562-572.

87 Chan, M.; Estève, D.; Fourniols, J.-Y.; Escriba, C.; Campo, E. Artificial Intelligence in Medicine 2012, 56, 137-156.

88 Rodgers, M. M.; Pai, V. M.; Conroy, R. S. IEEE Sensors Journal 2015, 15, 3119-3126.

89 Stoppa, M.; Chiolerio, A. Sensors 2014, 14, 11957-11992.

90 Aguirre, A.; Testa-Weintraub, L.; Banderas, J.; Haraszthy, G.; Reddy, M.; Levine, M. Critical Reviews in Oral Biology and Medicine 1993, 4, 343-350.

91 Preston, A.; Edgar, W. Journal of Dentistry 2005, 33, 209-222.

92 Kim, J.; Valdés-Ramírez, G.; Bandodkar, A. J.; Jia, W.; Martinez, A. G.; Ramírez, J.; Mercier, P.; Wang, J. Analyst 2014, 139, 1632-1636.

93 Mannoor, M. S.; Tao, H.; Clayton, J. D.; Sengupta, A.; Kaplan, D. L.; Naik, R. R.; Verma, N.; Omenetto, F. G.; McAlpine, M. C. Nature Communications 2012, 3, 763.

94 Gamella, M.; Campuzano, S.; Manso, J.; de Rivera, G. G.; López-Colino, F.; Reviejo, A.; Pingarrón, J. Analytica Chimica Acta 2014, 806, 1-7.

95 Schazmann, B.; Morris, D.; Slater, C.; Beirne, S.; Fay, C.; Reuveny, R.; Moyna, N.; Diamond, D. Analytical Methods 2010, 2, 342-348.

96 Guinovart, T.; Parrilla, M.; Crespo, G. A.; Rius, F. X.; Andrade, F. J. Analyst 2013, 138, 5208-5215.

97 Gonzalo-Ruiz, J.; Mas, R.; de Haro, C.; Cabruja, E.; Camero, R.; AlonsoLomillo, M. A.; Muñoz, F. J. Biosensors and Bioelectronics 2009, 24, 1788-1791.

98 Windmiller, J. R.; Bandodkar, A. J.; Valdés-Ramírez, G.; Parkhomovsky, S.; Martinez, A. G.; Wang, J. Chemical Communications 2012, 48, 6794-6796.

99 Yan, Q.; Peng, B.; Su, G.; Cohan, B. E.; Major, T. C.; Meyerhoff, M. E. Analytical Chemistry 2011, 83, 8341-8346.

100 Kudo, H.; Sawada, T.; Kazawa, E.; Yoshida, H.; Iwasaki, Y.; Mitsubayashi, K. Biosensors and Bioelectronics 2006, 22, 558-562.

101 Thomas, N.; Lähdesmäki, I.; Parviz, B. A. Sensors and Actuators B: Chemical 2012, 162, 128-134.

102 Yao, H.; Shum, A. J.; Cowan, M.; Lähdesmäki, I.; Parviz, B. A. Biosensors and Bioelectronics 2011, 26, 3290-3296.

103 Tierney, M. J.; Tamada, J. A.; Potts, R. O.; Jovanovic, L.; Garg, S.; Team, C. R. Biosensors and Bioelectronics 2001, 16, 621-629. 